\title{
La palabra de los represores y el problema de la verdad en Argentina: reflexiones a partir de los dichos de Eduardo "Tucu" Costanzo
}

\author{
The Perpetrator's Confessions and the Problem of Truth in Argentina; \\ around the Statements of Eduardo "Tucu" Costanzo
}

Valentina Isolda Salvi ${ }^{1}$

\section{RESUMO}

A partir de las declaraciones realizadas por el imputado, Eduardo Tucu Costanzo, en el marco de la causa Guerrieri I, primer juicio por crímenes de lesa humanidad en la cuidad de Rosario, Provincia de Santa Fé (2009-2010), este artículo se propone, por un lado, analizar lo efectivamente dicho por un represor que rompió el "pacto de silencio" y sus condiciones de posibilidad; y por otro lado, dar cuenta de los efectos que esa palabra provocó una vez que fue puesta en circulación, el modo en que fue tratada, el carácter que se le otorgó, los debate suscitados y los respuestas y reacciones generadas entre los actores sociales involucrados personal y políticamente en el proceso judicial en Rosario. El contenido de las declaraciones de Costanzo como los efectos y reacciones que provocó permiten plantear una serie de cuestiones más generales respecto del lugar que ocupan los represores y su palabra en los procesos memoriales así como sobre los sentidos sobre la concepción de verdad que la circulación pública de esta palabra condensa.

Palavras-chave: Memoria. Dictaduras militares. Argentina. Represores. Declaraciones públicas.

\section{ABSTRACT}

This article is focused on the declarations made by Eduardo "Tucu" Costanzo, a defendant in the Guerrieri I Case, the first trial for crimes against humanity held in the city of Rosario, which took place between 2009 and 2010. With the aim of contributing to the debate about the social, political and moral weight of the "first person" testimony of the perpetrators and the different senses of truth that this words produce, in this article I also analyze the repercussions and

\footnotetext{
1 Centro de Investigaciones Sociales-CONICET/Instituto de Desarrollo Económico y Social (IDES). Este artículo se realizó en el marco del proyecto PICT 2013-0299 "Las declaraciones públicas de represores: narrativas y conflictos en la memoria social sobre el terrorismo de estado en la Argentina". Agradezco a los/as colegas del PICT y del Núcleo de Historia Reciente del IDAES/UNSAM por sus valiosos comentarios. E-mail: valentinasalvi@hotmail.com
} 
controversies these declarations aroused among the social actors that were personally and politically involved in the judicial process in Rosario. For this purpose, I will provide a similarly brief description of the social conditions whereby these declarations were able to be made and heard, in order to examine the meanings and representations of the violence of the past that are brought into play once unknown (and horrific) aspects of the illegal repression are made public in the voice of one of its perpetrators.

Keywords: Memory. Dictatorship. Argentina. Perpetrators. Public Statements.

En el año 2006 se reinicia en Argentina un nuevo ciclo de juzgamiento a militares, policías y civiles acusados de violaciones a los derechos humanos, luego de la suspensión de los procesos penales que implicaron las sanciones de las leyes de Punto Final y Obediencia Debida en 1986 y 1987. ${ }^{2}$ Treinta años después de las leyes de impunidad, más de un millar de represores fueron condenados por crímenes de lesa humanidad, y otros 800 se encuentran procesados con causas en curso. En el marco de las audiencias orales de la causa conocida como Guerrieri I, primer juicio por crímenes de lesa humanidad de la Ciudad de Rosario, se realizaron las declaraciones indagatori as del personal civil de inteligencia (PCI), ${ }^{3}$ Eduardo "Tucu" Costanzo, de las que me ocuparé en este artículo. ${ }^{4}$ Costanzo es uno de los pocos represores que rompió el llamado "pacto de silencio" y que ratificó sus dichos extrajudiciales ante el tribunal federal que lo juzgó por delitos gravísimos en 2009 y $2010 .^{5}$

Los dichos de Costanzo se efectuaron en el marco jurídico de una declaración indagatoria. ${ }^{6}$ Si bien, según indica el Código Procesal Penal, el imputado tiene derecho a guardar silencio sin que implique presunción de culpabilidad en su contra, Costanzo habló in extenso durante las audiencias orales. En el plano de la imputación penal, las declaraciones

2 En junio de 2005, la Corte Suprema de la Nación declaró inconstitucionales estas leyes avalando la Ley 25.779, mediante la cual el Congreso había anulado las normas de impunidad en 2003. De este modo, quedó allanado el camino para que avanzasen los juicios por delitos de lesa humanidad.

3 Los grupos de tareas, llamados en la jerga represiva patotas, que operaron de manera directa en la represión ilegal estaban conformados por miembros de las fuerzas armadas y de seguridad y por civiles reclutados a tal fin o que militaban en organizaciones paramilitares de derecha.

4 El expediente no 131/2007 "Guerrieri, Pascual y otros s/ priv. ilegal de la libertad, amenazas, tormentos y desaparición física" (y acumulado № 42/09 "Amelong, J y otros) se tramitó ante el Tribunal Oral Federeal №2 a cargo de los jueces Otmar Paulucci, Beatriz Caballero de Barabani y Jorge Venegas Echagüe. Esta causa resolvió sobre las desapariciones de Jorge Novilo, Eduardo Toniolli, Miguel Ángel Tossetti, Carlos Rodolfo Juan Laluf, Marta María Benassi, Ana María Gurmendi, Oscar Daniel Capella, Fernando Dante Dussex, Stella Hildbrand de Del Rosso, Teresa Soria de Sklate, María Adela Reyna Lloveras, Héctor Pedro Retamar, Marta María Forestello, Raquel Negro y Edgar Tulio Valenzuela correspondientes a los Centros Clandestinos de Detención (CCD) Quinta de Funes, Escuela Magnasco y La Intermedia, y de Hilda Cardozo, Ariel Morandi y Susana Miranda para el CCD Fábrica Militar de Armas Domingo Matheu; y sobre los secuestros y tormentos a Jaime Dri, Carlos Novillo, Alejandro Novillo, Graciela Zitta, Emma Buna, Liliana Nahs de Bruzzone, Adriana Arce, Ramón Verón, Juan Rivero y Olga Moyano.

5 Entre los miembros de las fuerzas armadas y de seguridad que brindaron información se pueden nombrar a Rodolfo Peregrino Fernández ante la Comisión Argentina de Derechos Humanos de Madrid en 1983 y ante la CONADEP, Raúl Vilariño en 1984 para la Revista La Semana y la CONADEP, Omar Torres y Adolfo Salman ante la CONADEP en 1984, Gustavo Bueno ante el CELS en 1986, Adolfo Scilingo para el libro El Vuelo y el programa de televisión Hora Clave en 1995 y Víctor Ibánez en el programa televisivo Hadad \& Longobardi en el mismo año.

6 La declaración indagatoria es parte del derecho a defensa que tiene un imputado para descargar o aclarar los hechos de los que se le acusa. Según el Art. 296 del Código Procesal Penal no se puede requerir al imputado juramento de decir la verdad, ni se puede ejercer sobre él coacción para que declare. 
de Costanzo tuvieron efectos concretos sobre los hechos tratados en el juicio y sobre la situación de los co-imputados, y fueron valoradas por el tribunal, la fiscalía y las querellas como información provechosa para la producción de prueba jurídica como se reflejó en los alegatos y en la sentencia. No obstante ello, tanto el contenido de las declaraciones del represor como su posicionamiento personal respecto de los hechos que relató fueron objeto de reacciones, polémicas y debates entre los actores implicados en el proceso judicial en Rosario. Familiares, víctimas, abogados querellantes, fiscales, imputados, defensores oficiales y jueces interpretaron y valoraron sus afirmaciones con diferencias que dan cuenta de matices éticos, políticos y jurídicos. ${ }^{7}$

El propósito de este artículo es, por una parte, identificar y analizar lo efectivamente dicho por Costanzo y sus condiciones de posibilidad y de escucha; y por otra parte, dar cuenta de los efectos que esa palabra provocó una vez que fue puesta en circulación, el modo en que fue tratada, el carácter que se le otorgó, los debates suscitados y las respuestas y reacciones generadas entre los actores que fueron parte del juicio. Pues bien, el contenido de lo dicho por Costanzo en sede judicial (y fuera de ella) y los efectos y reacciones que esta palabra provocó permiten plantear una serie de cuestiones más generales respecto del lugar que ocupan los represores y su palabra en los procesos memoriales. ${ }^{8}$ En tal sentido, me interesa comprender, primero, cuál es el lugar que adquiere la palabra de los represores en las luchas por la memoria; y segundo, cuáles son los sentidos sobre la concepción de verdad que la circulación pública de esta palabra condensa. Por último, el análisis que me propongo realizar, a partir de los dichos de Costanzo, busca contribuir a una reflexión mayor sobre las declaraciones de represores en contextos de impunidad y de persecución penal y sobre las consecuencias que estos contextos producen en los planos de enunciación de la verdad sobre el pasado reciente en Argentina (FELD, 2016, p. 79).

Para la realización de este artículo se reunieron tres tipos de fuentes. En primer lugar, se consultaron las audiencias orales de la causa Guerrieri I disponibles en el Archivo Audiovisual de Juicios de Lesa Humanidad de Provincia de Santa Fé para identificar los dichos de Costanzo, enmarcarlos en el debate oral que tuvo lugar en el recinto y ponerlos en relación y contraposición con los dichos de los testigos y los co-imputados, los alegatos y la

\footnotetext{
7 Los otros imputados fueron los oficiales Pascual Guerrieri, Jorge Fariña, Juan Daniel Amelong y el PCI Walter Pagano. Junto con Costanzo, todos recibieron la pena de cadena perpetua.

8 La noción de represor, que es una categoría nativa de extendido uso académico, tiene en Argentina un sentido inequívoco, pues refiere de manera exclusiva a los miembros de las fuerzas armadas y de seguridad y personal civil de inteligencia que participaron en la represión ilegal y, por tanto, no resulta factible de ser aplicada a los miembros de las organizaciones armadas de izquierda. Además, en tanto categoría social tiene su historicidad y carga con la marcas de los procesos políticos y las luchas memoriales que buscaron denunciar y hacer visible el crimen de desaparición e identificar y enjuiciar a los responsables (SALVI, 2017)
} 
sentencia. En segundo lugar, se relevaron notas de prensa en medios locales y nacionales (Página/12, La Capital, Rosario/12 y El Eslabón) para rastrear las declaraciones extrajudiciales de Costanzo y las repercusiones y efectos que tuvieron antes, durante y después del juicio. ${ }^{9}$ Por último, se realizaron entrevistas a abogados querellantes, fiscales y defensores lo que permitió conocer los posicionamientos políticos, éticos y jurídicos que estos actores mantuvieron respecto de los dichos y la figura de Costanzo.

\section{El Represor y sus Primeras Declaraciones (Mediáticas)}

Oriundo de la Provincia de Tucumán, Eduardo Costanzo reconoció su participación en el Operativo Independencia bajo la conducción del general Acdel Vilas, causa que dice haber abrazado "con profunda y patriótica convicción" (Página/12, 13/4/1998). ${ }^{10}$ En agosto de 1976, quedó detenido acusado de robo en el mercado de concentración de frutas y verduras de la ciudad capital de esa provincia. Luego de ese episodio, Costanzo se trasladó con su familia a Rosario y, en julio de 1977, a través de la recomendación de un miembro de la patota, el capitán Marino González, ingresó al Batallón de Inteligencia 121 de esa ciudad. Durante el primer mes, Costanzo dijo que "estuvo de oyente", período durante el cual tuvo que "mirar cómo trabajaban, cómo operaban [...] hasta que vino el nombramiento". ${ }^{11}$ Hasta diciembre de 1978, según sus dichos, Costanzo fue parte de la patota que operó en cinco centros clandestinos de detención de la provincia de Santa Fé: La Calamita, Quinta de Funes, Escuela Magnasco, La Intermedia y Fábrica Miliar de Armas Domingo Matheu. ${ }^{12}$

En tanto $\mathrm{PCl}$, Eduardo Costanzo no era un oficial ni un suboficial de carrera, ni miembro de la familia militar, sino que fue reclutado por el Ejército para formar parte de la patota. Los $\mathrm{PCl}$ no contaban con formación militar o policial, eran más bien mano de obra flexible con capacidad de acción descentralizada y discrecional, reclutada en las

9 Pagina/12 y Rosario/12 son periódicos con un perfil ideológico de izquierda y están directamente vinculados a la causa de los derechos humanos, el primero tiene una tirada nacional y el segundo es un diario local de la Ciudad de Rosario. El Eslabón es un semanario local de perfil crítico y alternativo a los medios dominantes en sintonía con la acción de los organismos de derechos humanos. Por el último, La Capital es el periódico tradicional de la Ciudad de Rosario con más 150 años de perfil de centro y centro derecha.

10 A comienzos de 1974, durante el tercer gobierno peronista, el Ejército Revolucionario del Pueblo (ERP) abrió en la zona selvática del sudoeste de provincia norteña de Tucumán un frente militar rural. El 5 de febrero de 1975, la presidenta Isabel Martínez de Perón firmó el decreto 261 que ordenaba "ejecutar las operaciones militares que sean necesarias a efectos de neutralizar y/o aniquilar el accionar de los elementos subversivos que actúan en la provincia de Tucumán". Con ello, comenzó el Operativo Independencia bajo la conducción del general Acdel Vilas, quien implementó un "Plan Táctico" que se basó en el combate directo contra ERP en el monte y en la realización de acciones clandestinas e ilegales en los pueblos de la zona y en la ciudad de San Miguel de Tucumán. Esta forma de represión ilegal constituyó un ensayo de lo que, a partir de 1976, se extendió hacia todo el territorio nacional bajo la forma de terrorismo de Estado.

11 Archivo Audiovisual de Juicios de Lesa Humanidad, 2015, Hoja 2 17/02/2010, recuperado de https://www.santafe. gov. ar/aajlh/item.php?id=127844\&cod=1dab22aa08b21f40e027a07af79455d0

12 La represión ilegal en la Ciudad de Rosario estuvo bajo control del Segundo Cuerpo de Ejército cuyo comandante fue el general Leopoldo Galtieri. La patota en que participó Costanzo dependía del Batallón del Inteligencia Militar 121 de esa ciudad (ÁGUILA, 2018). 
universidades, fábricas, barrios o pueblos donde debían hacer tareas de infiltración y ser informantes de las fuerzas de seguridad. Muchos de estos cuadros ya formaban parte de grupos paramilitares durante el gobierno de Isabel Martínez de Perón. Ese fue el caso de Eduardo Costanzo, quien fue reclutado para ser parte de grupos comandos que actuaron durante el Operativo Independencia en la provincia de Tucumán. Luego de su paso por la patota del Batallón de Inteligencia 121 de Rosario, los últimos años de la dictadura, Costanzo tuvo que cumplir una condena por homicidio fruto de una riña callejera. Ya iniciada la democracia, Costanzo contó haber trabajado como "mano de obra desocupada" para el senador justicialista, Luis Rubeo. ${ }^{13}$

Al final de la dictadura, Costanzo ya era conocido por su apodo: "el tucu". Dar cuenta de la identidad de los represores fue una acción tempranamente emprendida por los sobrevivientes y las organizaciones de derechos humanos. La confección de listas con nombre, apellido y apodo, la construcción de los organigramas de las cadenas de mandos, la identificación de los miembros de las patotas que operaban en cada CCD en base a testimonios de sobrevivientes permitió la creación de un acumulado de información sobre la identidad de los represores que sirvió como insumo para las tareas de la CONADEP y la posterior persecución penal. ${ }^{14}$ En el libro Recuerdo de la muerte, Jaime Dri, único sobreviviente del CCD Quinta de Funes, se refiere a Costanzo como "el tucu: con la cachaza tucumana. Viejo. Experimentado. Ojo, es observador" (BONASSO, 2001, p. 238). En 1986, Gustavo Bueno, otro PCl de la patota, en una declaración ante el CELS nombró a Eduardo Costanzo por el nombre de revista que aparecía en su Legajo Militar: Ernesto Castro. Según la declaración de Gustavo Bueno, el "tucu" "era uno de los que cuidaba el sector donde estaban los detenidos" (Alegato Querella H.I.J.O.S., p. 79).

Durante las audiencias orales, los testigos sobrevivientes mencionaron a Costanzo en varias oportunidades: Jaime Dri declaró que el Tucu era una de las personas a las que veía frecuentemente en lugares en los que estuvo cautivo. Olga Moyano dijo que, en el CCD Fábrica Militar de Armas Domingo Matheu, había una persona a la que le decían "el Tucu" quien a veces les llevaba comida. Ramón Verón y Juan Antonio Rivero también lo mencionaron entre los represores que estaban en ese CCD. Por último, Adriana Arce lo relacionó una de las personas que la siguió en el ómnibus, momentos antes a su secuestro,

13 En los años de la transición democrática se llamaba "mano de obra desocupada" a los ex miembros de grupos de tareas. Costanzo declaró en RADIO LT8 que, como parte de esos "trabajos" para Rubeo, colocó un aparato explosivo a su contrincante electoral en 1987, el diputado radical Carlos Perretta (Rosario/12, 12/01/2008).

14 En agosto de 1983, antes de concluir la dictadura, los organismos de derechos humanos formaron una comisión técnica de recopilación de información sobre desaparecidos, CCDs y represores (CRENZEL, 2008, p. 55). En 1986, el Centro de Estudios Legales y Sociales (CELS) publicó un informe titulado "Terrorismo de Estado. 692 responsables" que contenía un listado organizado a partir de la cadena de mando en cada dependencia militar o policial (CELS, 1986). 
por su acento del norte argentino. Por su parte, Costanzo dijo que era chofer, que manejaba cuando se hacían las "detenciones" y que se quedaba "cuidando a los presos cuando salían a detener". También dijo que nunca "detuvo", ni "torturó", ni "interrogó", ni "mató" a nadie; aunque reconoció su participación en el ocultamiento de los cuerpos de los desaparecidos, sea inhumando clandestinamente cuerpos o envolviéndolos con frazadas y amarrándolos para ser arrojados desde aviones al mar.

Costanzo alcanzó notoriedad pública local cuando, en junio de 1992, Rosario/12 publicó una nota en la que el represor dio detalles sobre la frustrada Operación México ${ }^{15}$, y explicó el modo en que fueron asesinadas las personas que habían estado secuestradas en la Quinta de Funes y, luego, arrojadas al mar desde aviones. En esa entrevista, Costanzo brindó los nombres completos y apodos de algunos de los miembros de la patota que eran desconocidos hasta ese momento. ${ }^{16}$ Al igual que el marino Adolfo Scilingo, aunque tres años antes, Costanzo se acercó a un medio de prensa a fin a la causa de los derechos humanos para hablar sobre lo sucedido durante el terrorismo de Estado. ${ }^{17}$ Costanzo habló también de los llamados "vuelos de la muerte", aunque a diferencia de Scilingo, no dio testimonio sobre su participación directa en ellos. ${ }^{18}$

Respecto de las condiciones de enunciación y escucha específicas en las que se produjeron las primeras declaraciones públicas de Costanzo en los medios de comunicación entre 1992 y 1998 - período en el que estuvo cerrada la vía judicial por la vigencia de las leyes de Punto Final y Obediencia Debida-, resulta necesario destacar tres cuestiones que permiten analizar el contexto social y político en el que se efectuaron. En primer lugar, el escenario en el que tuvieron lugar fue el de los medios de comunicación -periódicos, televisión, revistas, libro periodístico- y, por tanto, el principal interlocutor fue un periodista y estaba dirigido a un tipo particular de audiencia según el medio de comunicación de que se tratara. De modo que quedó, por un lado, en manos de ese medio de prensa y de su posición político-ideológica la potestad de enmarcar y tematizar los dichos de Costanzo; y

15 La Operación México, ideada por el general Galtieri y organizada por el Batallón de Inteligencia 121, tuvo por objetivo infiltrar y asesinar a la conducción de Montoneros exilida en ese país. Para ello, el militante montonero secuestrado, Tucho Valenzuela, fue obligado a viajar a México para que delatase a los dirigentes bajo amenaza de matar a su mujer, Raquel Negro, también secuestrada y embarazada mellizos, y al hijo de ella. Junto con Valenzuela viajaron a México los represores Jorge Fariña, Juan Daniel Amelong y Juan Andrés Cabrera y otro secuestrado, Carlos Laluf. Una vez en México, Valenzuela tomó contacto con la organización y denunció públicamente la operación.

16 Costanzo dio una entrevita a la Revista Gente el 9 de julio de 1992, participó de un programa de Canal 5 de Rosario con el periodista Alberto Gonzalo y en dicieimbre de 1997, el periodista Carlos del Frade lo entrevistó para su libro El Rosario de Galtieri y Feced.

17 En marzo de 1995 se publica el libro El vuelo de Horacio Verbitsky basado en un entrevista a Adolfo Scilingo quién cuenta sobre su participación en los vuelos de la muerte en los que se arrojaban a detenidos-desaparecidos vivos que habían estado secuestrados en la ESMA.

18 Costanzo alcanzó notoriedad en los diarios nacionales cuando, en abril de 1998, se hizo pública una carta que le había enviado a Raúl Topa, vice gobernador de Antonio Bussi en la provincia de Tucumán, en la que lo involucraba en actividades represivas en esa provincia (Página/12, 14/4/1998). 
por otro lado, en los mecanismos propios del formato periodístico -ya sea prensa, televisión o periodismo de investigación- la inclusión de su palabra dentro de la construcción de un discurso verosímil sobre el pasado con sus propios patrones de autenticidad, credibilidad y validez.

En segundo lugar, dado el escenario mediático en el que la palabra de Costanzo tuvo lugar, los familiares de las víctimas y los sobrevivientes no contaban con instancias o mediaciones institucionales para poder controvertir sus dichos y/o encauzar sus demandas políticas o necesidades personales frente a lo que escuchaban y los afectaba directamente. Lo que implicó, en muchos casos, que la respuesta fuera la publicación de comunicados o la realización de conferencias de prensa (también en los medios de comunicación) para hacer público un descargo o marcar posiciones políticas al respecto de lo dicho por el represor. ${ }^{19}$

En tercer lugar, la información aportada por Costanzo no se encauzó a través de mecanismos institucionales que junto con otros hechos ya probados en la justicia o reconstruidos a través del trabajo de la CONADEP confluyeran hacia una verdad esclarecedora sobre lo sucedido en los CCDs y con los desaparecidos en Rosario. En esas condiciones de enunciación, la corroboración de la información brindada por Costanzo quedó en manos de los organismos de derechos humanos y sobrevivientes. Esto muestra al mismo tiempo el interés que tenían algunos familiares por obtener información sobre la suerte corrida por sus seres queridos y la situación de vulnerabilidad en que la que se encontraban ante la ausencia de mecanismos institucionales que permitiesen encauzar la búsqueda de verdad. ${ }^{20}$

Estas particulares condiciones de enunciación y de escucha -que tuvieron lugar especialmente en la década del 90' cuando los represores estaban protegidos por las leyes de impunidad- redundaron en una forma específica de presencia de su palabra la vida pública. ${ }^{21}$ En esos años, la lógica espectacular y exhivitiva de los medios de comunicación

19 Otro tipo de respuesta fue cursar una denuncia ante la justicia en los casos en que estas declaraciones fueron lesivas y amenazantes para las víctimas. Por ejemplo, el ex Jefe de la Policía de la Provincia de Buenos Aires, Miguel Etchecolatz, fue condenado a tres meses de presión en suspenso y a hacer un curso de derechos humanos por haber calumniado e injuriado al diputado Alfredo Bravo (quién había sido secuestrado y torturado por la patota a su cargo en 1977) en un programa de televisión en 1997. Alfredo Astiz fue también condenado a tres meses en suspenso tras la reivindicación de la tortura que realizó en una entrevista publicada en la Revista Tres Puntos en 1998.

20 En 1995, el CELS, Abuelas de Plaza de Mayo y Madres de Plaza de Mayo-Línea Fundadora procuraron estrategias para acercarse a Scilingo en busca de información (VARSKY, 2011, p. 71-72). Costanzo, por su parte, citó a Cecilia Nazábal y Alicia Gutiérrez, en el mercado de concentración de frutas y verduras al poco tiempo de que fuese publicada la nota en Rosario/12 hacia 1992. Ambas referentes de derechos humanos en Rosario, a pesar del interés por obtener más información sobre sus familiares desaparecidos, no asistieron a la cita por los riesgos que podrían correr. (Nadia Schujman, abogada de la Querella H.I.J.O.S., entrevista realizada 17/7/2016)

21 A las ya mencionadas declaraciones mediáticas del capitán de corbeta (R) Adolfo Scilingo, en 1995, se agregan las del ex sargento Víctor lbáñez en el programa Hadad \& Longobardi el 24 de abril, del capitán (R) Héctor Vergéz en el programa Hora Clave el 23 de marzo, del sargento $(\mathrm{R})$ de la policía federal Julio Simón en el Noticiero de ATC el de 1 de mayo y en Telenoche Investiga el 2 y 3 de mayo, del ex almirante Emilio Massera el 7 y 10 de agosto en Hadad \& Longobardi y Hora Clave respectivamente; y del ex comisario Miguel Etchecolatz en el programa de televisión Hora Clave el 25 de agosto de 
contribuyó a la conformación de dos supuestos a partir de los cuales se tendió a valorar la palabra de los represores. Ambos supuestos, si bien surgieron del modo en que los medios tematizaron y presentaron las declaraciones de los represores durante la década del 90', se convirtieron una suerte de sentido común sobre la naturaleza de esta palabra que tendió a imponer ciertos modos de interpretarla y valorarla socialmente que buscan ser revisados en este artículo.

El primer supuesto tendió a considerar como una confesión las declaraciones públicas de represores cuyo carácter revelador era dado por real sin cotejar si lo efectivamente dicho constituía información conocida o no (SALVI, 2016). Este supuesto se apoya en una contradicción inmanente a la figura de la confesión: por su carácter performativo el acto de habla se convierte en una acción en sí misma y tiene la capacidad de construir un efecto de verdad por el solo hecho de hablar (GRÜNER, 1995, p. 31). De modo que, durante la década del 90, cuando los represores hablaron en público, los medios de prensa sobrevaloraron los acto de habla más allá del contenido de lo dicho. El segundo supuesto, por su parte, planteaba que una verdad más verdadera que la producida por los testigos, por los sobrevivientes, por las CONADEP o que los diversos juicios provendría de los mismos represores (FELD, 2016). Este segundo supuesto busca ser revisado a la luz de análisis de los dichos de Costanzo, su valoración y sus efectos en el marco del juicio que se celebró en Rosario.

Mostrar el carácter contingente de estos supuestos como modos de construcción y valoración de la palabra de los represores, permite abrir líneas de análisis que permitan desesencializar su palabra para aproximarla a su historicidad, es decir, a los modos en que es producida, valorada, demandada y escuchada socialmente para comprenderla como un factor activo que incide en las luchas y construcción de las memorias en la Argentina. ${ }^{22}$ Desesencializar esta palabra significa diferenciar diversos planos de enunciación de la verdad sobre el pasado en los que las declaraciones de los represores se articulan y a través de los adquiere significación social, y no suponer una relación con la "Verdad" como una condición inmanente y natural derivada de la posición de victimario y de sus actos de habla.

1997 y las afirmaciones del capitán de fragata (R) Alfredo Astiz en enero de 1998 en la revista Tres Puntos (FELD, 2001, 2016).

22 En términos conceptuales, retoma las consideraciones de Joan W. Scott (2001) sobre la necesidad de dar historicidad a la noción de experiencia y las transpolo al análisis de la palabra de los perpetradores. Al igual que la experiencia que es considerada por el conocimiento positivista como una evidencia, reflejo de lo real y punto de partida de la explicación (SCOTT, 2001, p. 47), la palabra de los perpetradores (por el hecho de tratarse de quienes participaron de la escena del crimen y están vivos para contarlo) es significada en términos esencialistas como acceso a la verdad o como una verdad en sí misma. Dar historicidad (des-esencializar) esta palabra implica no solo mostrar el carácter construido de estas formas de valoración sino también dar cuenta de las procesos y disputas en los que se escucha, valora e interpreta dicha palabra. 


\section{"Yo Como Testigo y Denunciante"}

En mayo de 2004, luego de la reactivación de la causa Guerrieri I, Costanzo fue detenido. ${ }^{23}$ Este hecho abrió una nueva seguidilla de declaraciones en los medios y en los tribunales federales de la Provincia de Santa Fe. ${ }^{24}$ En el marco de las audiencias orales, Costanzo realizó tres ampliaciones de indagatorias y aceptó preguntas de la fiscalía, las querellas, el tribunal y de su propio de defensa y rechazó las de las defensas de los otros imputados. En un clima de hostilidad con sus ex -compañeros y dadas las denuncias de amenazas de parte de Juan Daniel Amelong, un gendarme sentado en la sala de audiencia separaba físicamente el lugar donde se ubicaba Costanzo de las sillas del resto de los imputados. $^{25}$

Respecto de los motivos que lo impulsaron a hablar, y a pesar de haber recibido amenazas de muerte de parte de sus ex -compañeros, Costanzo dijo que era un "chivo expiatorio" de sus superiores, a quiénes decidió "denunciar" porque lo "quieren incluir" en hechos que fueron "ejecutados" por ellos. ${ }^{26}$ Él se presenta ante el tribunal como "testigo" y "denunciante" y le agradece la oportunidad que le brinda de "poder decir toda la verdad".

\footnotetext{
Yo me declaro totalmente inocente de todos estos hechos de los que se me acusa. Tal es así que yo me presenté espontáneo a la justicia para que se me investigara. Soy el primer denunciante y testigo que dio la cara sin temer a nadie cuando todos se callaron. ${ }^{27}$
}

Desde este lugar de enunciación, Costanzo describe su participación en los hechos poniendo la distancia de un testigo que denuncia lo que sabe, lo que vio, lo que escuchó. No utiliza la figura de la confesión para enmarcar sus declaraciones, pues esto implicaría el reconocimiento del yo como partícipe, sino que lo hace desde la figura de la denuncia que implica la posición de un yo que decidió no callar lo que sabe y acusar a otros, especialmente a sus superiores y al Ejército. Sin embargo, el relato Costanzo fue posible

23 La primera causa que se reabrió fue la de la Quinta de Funes cuando el juez Omar Digerónimo en 2003 declaró la inconstitucionalidad de las leyes de impunidad. Hasta llegar al juicio oral, la causa pasó por tres jueces federales: Omar Digerónimo, Carlos Vera Barros y Germán Sutter Schneider.

24 Costanzo efectuó, el 11 de enero de 2008, una extensa entrevista por Radio LT8 y en febrero del mismo año, otra en Rosario/12

25 Costanzo fue patrocinado por un defensor oficial, Germán Artola, luego de haber desistido de la defensa privada de Osvaldo Gandolfo quién le recomendaba que no declarase y se negaba a solicitar ampliaciones de indagatorias durante la etapa procesal. (Entrevista a Germán Artola, 14/05/2016).

26 Otras casos como los del Gendarme Jorge Torres, el del PCl Gustavo Bueno o del Cabo Miguel Ángel Pérez muestran que las fisuras en el pacto de silencio y el resquebrajamiento del discurso que niega u oculta la desaparición de personas se produciría más frecuentemente entre los cuadros bajos de la estructura, entre aquellos que tienen menor organicidad o que se dieron de baja de la fuerza.

27 Archivo Audiovisual de Juicios de Lesa Humanidad, 2015, Hoja 3 21/09/2009, recuperado de https://www.santafe.gov.ar/aajlh/item.php?id=124850\&cod=3c21ebc5d21b1 b50198b8a0f03534702 
porque se trata de alguien que estuvo allí, en la escena del crimen, y estuvo allí porque fue parte del grupo que cometió los crímenes.

No obstante su probada participación en los hechos, Costanzo no expresa arrepentimiento en términos personales, ni confiesa remordimiento, ni tiene mala conciencia por los hechos de los que fue parte (JANKÉLÉVITCH, 1987). Costanzo no se presentó, ante el tribunal, como un conciencia atormentada con necesidad de confesar los horrores que fue capaz de cometer, sino que mostró el distanciamiento moral y la indiferencia de quién siente que estaba trabajando. En efecto, Costanzo buscó no implicarse o inculparse, y por eso se ubicó en la posición de un subalterno que cumplía órdenes y no tomaba decisiones.

Lo novedoso de las declaraciones de Costanzo es que no reproduce el discurso, común entre los oficiales, de la guerra antisubversiva librada contra el enemigo interno en defensa de la nación (SALVI, 2012). No hay rastros de este discurso político de los vencedores o salvadores tan repetido por los acusados a la hora de justificarse en el marco de los juicios cuando hacen uso de la palabra. De un modo descriptivo y literal, Costanzo evoca ciertas imágenes que denotan una marcada insensibilidad y cinismo que perturba a quién lo escucha. También utiliza expresiones del habla popular que connotan su condición de clase y reproduce el lenguaje de los CDD: ese idioma displicente y rudo, lleno eufemismos, ironías, incluso, chistes crueles que deshumaniza a las víctimas y cosifica a los victimarios.

En términos generales, tras la declaraciones de Costanzo se deja entrever el funcionamiento de un aparato represivo que combinaba rutina operativa, clandestinidad y libertad de acción. La división de tareas en el funcionamiento del grupo operativo, el carácter compartimentado de la información entre los represores, el modo improvisado de la ejecución de las acciones y el uso de violencia extrema en las conductas delictivas se hacen evidentes en los hechos que describió. Además aparece un elemento central del funcionamiento de la maquinaria represiva: el convencimiento de que las tareas que él cumplió -en calidad de subordinado y fruto de la división de trabajo- eran inofensivas, diluyendo su responsabilidad en los superiores y en el funcionamiento cotidiano del CCD.

A diferencia de Costanzo, los otros cuatro imputados en la causa mantuvieron silencio o tomaron la palabra para negar o reivindicar políticamente los hechos. Con un lenguaje de denuncia y acusación hacia sus ex-camaradas, Costanzo también buscó acercarse al ethos de los organismos de derechos humanos y seducir a los querellantes en la causa para que pidan una pena menor que al resto de los imputados por el hecho de haber hablado. Por otra parte, Costanzo guardaba esperanzas de quedar beneficiado por la figura del 
arrepentido que existe en el caso de la ley de estupefacientes en pos de una reducción de penas; figura no contemplada en el Código Penal.

Ahora bien, ¿qué fue lo que dijo Costanzo en las audiencias orales? A fin clarificar cuáles fueron sus aportes, clasificaremos la información brindada en cinco tipos: 1) identificó a miembros de la patota; 2) describió las circunstancias en las que fueron asesinadas diversos grupos de detenidos-desaparecidos; 3) señaló el lugar en el que fueron inhumanos clandestinamente restos de desaparecidos; 4) precisó el modo en que se efectuaron secuestros; y 5) contribuyó con información para la restitución de la identidad de una hija de desaparecidos.

Una de las características fundamentales de la desaparición de personas fue el ocultamiento y la invisibilización del crimen y de los criminales. Si bien con el testimonio de los sobrevivientes y con la información recabada por los organismos de derechos humanos sobre las cadenas de mandos y los destinos asignados a los miembros de las fuerzas de armadas y de seguridad, fue posible progresivamente vincular los apodos de los represores con los nombres y grados de los militares y policías. Sin embargo, no se conoce la identidad de todos los represores. Ese rompecabezas siempre se está completando. Por eso, en este punto, el aporte de Costanzo fue significativo dado que identificó con nombre, apellido y apodo a sus ex -compañeros de la patota, dando cuenta de la función que cumplían dentro del grupo represivo y señalando algunos de los hechos en los que estuvieron involucrados. Uno por uno, nombró a 22 de sus ex camaradas, les atribuyó a cada uno una función y los vinculó a hechos criminales determinados. Veamos solamente algunos casos, sobre Rubén Alcuri, alias el Petiso Aguilar, dijo:

[...] es el que estuvo en el secuestro y tortura de Adriana Arce, es el que tenía la orden de sacar fotografías a todos los detenidos antes de ser arrojados al mar porque era el fotógrafo que tenía el destacamento. Habría que preguntarle si tiene Sobre el Comisario (r) Rodolfo Isach, alias Agustín: los negativos. Hoy está encargado de agencia de seguridad acá en Rosario. ${ }^{28}$

[...] es el que le dio muerte a los 14 detenidos de La Intermedia, finca de los Amelong, lo hizo con una ametralladora con silenciador pegándole dos tiros en el corazón a cada uno con el coronel Guerrieri, el teniente coronel Fariña y teniente coronel Amelong. ${ }^{29}$

Sobre Juan Carlos Bossi, alias Julio Balanchar:

28 Archivo Audiovisual de Juicios de Lesa Humanidad, 2015, Hoja 3 21/09/2009, recuperado de https://www.santafe. gov. ar/aajlh/item.php?id=124850\&cod=3c21ebc5d21b1b50198b8a0f03534702

29 Archivo Audiovisual de Juicios de Lesa Humanidad, 2015, Hoja 4 21/09/2009, recuperado de https://www.santafe.gov. ar/aajlh/item.php? 
[...] es el que se ponía la chaquetilla de médico y los inyectaba a los detenidos para adormecerlos, y les ponía la llamada corbatita. Yo no sabía lo que era esa corbatita hasta un día pude averiguar con un ex compañero de trabajo, que me dijo que consistía en una goma de esa que usan para sacar sangre, que le ponían en el cuello hasta que quedaban muertos. Bossi es uno de los integrantes de los vuelos de la muerte. ${ }^{30}$

A pesar de lo extenso de la lista de represores identificados -algunos de los cuales no se conocían-, no se puede tener certeza de que Costanzo haya nombrado de manera efectiva a todos los que conocía o haya descripto todos las acciones criminales cometidas por ellos. ${ }^{31}$ Las identificaciones realizadas le valió la respuesta de los co-imputados, quienes ampliaron sus declaraciones indagatorias para desacreditar sus dichos aludiendo alevosía personal, desconocimiento, imprecisión, calumnia y problemas de orden psíquico.

En segundo lugar, Costanzo describió y dio a conocer con detalles hechos que sólo él -y los otros acusados en el juicio $u$ otros represores- estaban en condiciones de conocer dado que no hay víctimas sobrevivientes. Me refiero a los asesinatos. Tal como sostiene Calveiro (1998, p. 38), se trata de una de las partes del proceso de desaparición "que más se desconocen", debido al "secreto que rodeaba a los procedimientos de traslado". Aunque sí se sabe, por los testimonios de las víctimas, que eran momentos que "estaban rodeados de una enorme tensión y violencia" (CALVEIRO, 1998, p. 38) tanto por lo que los propios secuestrados intuían que podía sucederles como por "las infidencias de guardias que no soportaban la presión a la que ellos mismo estaban sometidos" (CALVEIRO, 1998, p. 51).

Costanzo habló sobre las circunstancias y el modo en que fueron asesinadas 13 personas en La Intermedia. ${ }^{32}$

\begin{abstract}
Se hizo un asado a las 12 del día, con los que había más los gendarmes, las chicas y los presos que estaban ahí porque habían hecho correr la bolilla que se iba en libertad María. La chica que hacía mucho tiempo que estaba allí, la chica que hacía de cocinera, que cocinaba para ellos, para nosotros para todos. Y a la noche llegamos nosotros, a festejar, era una fiesta. Han puesto una mesa, allí en el chalet. [...] Nos hicieron dejar el auto a un kilómetro, llegamos a pie, sin armamento. [...] estábamos todos allá esa noche. Estuvimos festejando, comiendo esa noche, todos, alegremente. Hasta que llegó la hora del whisky, hasta que Fariña le dice a Sergio [...] "andá, traé dos botellas de whisky que tengo en el auto". El otro va y trae las dos botellas, sabía cual era una y cual era la otro, pone donde estábamos nosotros una botella de whisky y en la otra parte, donde estaban los montoneros, otra botella. [...] la habían preparado en Campo de Mayo. Que estos chicos cuando tomen el whisky iban a entrar a llorar o se morían o se dormían. La primera en agarrarle eso que
\end{abstract}

30 Archivo Audiovisual de Juicios de Lesa Humanidad, 2015, Hoja 4 21/09/2009, recuperado de https://www.santafe. gov. ar/aajlh/item.php?id=125687\& cod=4062c6244f7547dd64e247f9ebd9d5e34

31 También nombró a Pascual Guerrieri, Francisco Scilabra, Carlos Isach, Ariel López, Carlos Sfulcini, Alberto Pelliza, Ricardo Ródriguez, Walter Roscoe, Gustavo Bueno, Juan Andrés Cabrera, Jorge Teves, Ariel Porra, Walter Pagano, Mario Vera, Juan Daniel Amelong, Jorge Fariña, Jorge Pérez Blanco, Marino González, Joaquín Gurrera, el gato Andrada y Sebastián alias Filtro.

32 Esa noche fueron asesinados Eduardo Toniolli, Fernando Dussex, Jorge Novillo, Stella Hilbrand de Del Rosso, Carlos Laluf, Marta María Benassi, Miguel Ángel Tosseti, Oscar Capella, Ana María Gurmendi, Héctor Retamar, María Reyna Lloveras, Teresa Soria de Sklate y Marta María Forestello. El cuerpo sin vida de Raquel Negro fue llevado esa misma noche a La Intermedia en un auto. 
tomaron cuando hicimos un brindis, nosotros con el whisky bueno de allá y ellos con el whisky ese, se largó a llorar la María, la llevaron a una habitación a atenderla. De ahí no la volví a ver más a María yo. Y los otros no quisieron tomar, algo sintieron que no quisieron tomar. Novillo y Tonioli dejaron el vaso, estaban al lado mío parados. [...] Cuando llega el coronel Guerrieri por la ventana vestido de verde con un fal colgado al hombro y le dice que el general Jáuregui estaba allá, en la casita esa. "Va a hablar uno por uno con Uds., no le hablen de Perón porque Perón lo tuvo en cana a él" les dice. [...] Después venía un gendarme y los llevaba uno por uno. Fulano y nosotros se lo entregábamos y se lo llevaban. Anda te llaman y lo acompañaba el gendarme y lo llevaban hasta la casita. [...] Y en la pieza estaban Rodolfo Isach, Fariña, Guerrieri y Amelong, son los cuatro que mataron a los 14. Isach sale después del hecho, que ha llegado el coronel Pozzi, sale con la ametralladora en la mano, y le dice, "vio mi coronel cómo se mata gente". [...] Uno más o menos sospechaba en la comida cuando iban llevando uno por uno ya no quedaba nadie. Y no volvían. Y después nos hacen quedar en la pieza de la casita donde estaban Fariña, Guerrieri, Amelong y Isach. Cuando nos hacen entrar dentro, y encontramos a la vueltita de la salita, así yendo para la pieza por el pasillo a los 14 todos amontonados totalmente desnudos muertos. De ahí nos ordenan que los saquemos a la salita uno por uno, le tapemos, con pedacito de trapo los dos agujeros que tenían en el corazón y los saquemos afuera y ahí los envolvían con colchas, estaban todos esperando ahí, estaban todos trabajando ahí, los envolvían con colchas y los cargaban a un camión que estaba parado ahí, un Mercedito $608 .{ }^{33}$

Si bien se tenía conocimiento que La Intermedia había sido el último destino del grupo de detenidos que habían pasado por la Quinta de Funes y la Escuela Magnasco, gracias al testimonio de Jaime Dri, único sobreviviente. ${ }^{34}$ Sin embargo no se conocían los detalles sobre el modo que fueron asesinados ni quienes fueron los responsables directos. Por eso, desde el punto de vista de la reconstrucción jurídica, el relato de Costanzo brindó detalles todos ellos estremecedores y horrorosos- que, si bien no pueden ser chequeados, fueron considerados como datos en el nivel de indicio para la construcción de prueba jurídica.

Ahora bien, desde el punto de vista sociológico, el relato de Costanzo deja entrever la forma de organización interna de los grupos de tareas y el modus operandi durante los llamados traslados. ${ }^{35}$ Respecto de los grupo de tareas, Costanzo menciona, por un lado, que estaban "todos allá esa noche" de modo de lograr el involucramiento de un número amplio de represores en la acción criminal; y por otro lado, describe la división de tareas y fragmentación entre quienes mataban, quienes -como los gendarmes- los buscaban para llevarlos a su destino final a unos metros en "la casita" y quienes se los "entregaban" a estos últimos -grupo en el que se encontraba Costanzo-. Uno y otra, el involucramiento colectivo y la segmentación de funciones producen el mismo efecto de licuación de la responsabilidad

33 Archivo Audiovisual de Juicios de Lesa Humanidad, 2015, Hoja 10 21/09/2009, recuperado de https://www.santafe. gov. ar/aajlh/item. php?id=141770\&cod=4e2557607ff3a64d4f38bc2531 a3ea5f

34 Jaime Dri fue secuestrado en Uruguay en diciembre de 1977 y trasladado a la ESMA. Luego fue enviado a la Quinta de Funes, a la Escuela Magnasco y a La Intermedia. Y de allí nuevamente a la ESMA. Logra escaparse de sus captores cuando fue enviado a la frontera con el Paraguay para que señalara a otros compañeros.

35 La utilización de los dichos de los represores como fuente sociológica debe ser problematizada y cotejada con otras fuentes disponibles pues existe la posibilidad de mentiras deliberadas (ÁGUILA, 2008, p. 28) En este caso, analizaremos los elementos que aparecen en las declaraciones de Costanzo y que se repiten en otras fuentes y otros casos que dan cuenta de la experiencia y la agencia de los perpetradores. 
moral de los victimarios. También Costanzo menciona que la plana mayor del Segundo Cuerpo de Ejército participó en la masacre, tanto su Subcomandante, el general Luciano Jáuregui como el jefe del Batallón del Inteligencia 121, el coronel Edgardo Pozzi. Lo que muestra que en los momentos de los asesinatos y de desaparición de los cuerpos -la parte del proceso represivo de mayor tensión- estaban presentes los altos mandos de las fuerzas armadas.

Sobre el modus operandi, Costanzo refiere al clima de tensión y ocultamiento que rodeó a este procedimiento criminal. Primero, dice que los miembros subalternos de la patota tuvieron que dejar los autos a un kilometro de distancia y dirigirse a La Intermedia "sin armamento". Segundo, hace referencia al cuidado de los represores por ocultar la muerte que esperaba a los secuestrados como estrategia para evitar toda forma de resistencia (CALVEIRO, 1998, p. 50). En este caso, el ocultamiento o disimulo de la masacre que estaba planificada se llevó adelante por medio de una estrategia absolutamente perversa: la puesta en escena de una fiesta dedicada a María de quién "habían hecho correr la bolilla que se iba en libertad". ${ }^{36}$ En tercer lugar, se trata del adormecimiento de los secuestros por medio de una inyección y, en este caso, de un whisky que lo habían "preparado en Campo de Mayo". Como explica Calveiro (1998, p. 38) "la aplicación del somnífero arrancaba al prisionero su ultima posibilidad de resistencia pero también sus rasgos más elementales de humanidad: la conciencia y el movimiento".

En tercer lugar, Costanzo mencionó el lugar en que fueron inhumados de manera clandestina cuerpos de desaparecidos. En el predio del Ejército Argentino ubicado en la Localidad de San Pedro señalado por Costanzo, el Equipo Argentino de Antropología Forense (EAAF) encontró ocho cuerpos en 2010, de los cuales fueron identificados los restos de María Esther Ravelo, una detenida-desaparecida no vidente que fue secuestrada en setiembre de 1977 y Gustavo Pon, un joven maestro oriundo de Gualeguaychú secuestrado en junio de 1977.

En cuarto lugar, Costanzo identificó a los represores que llevaron a cabo el secuestro de los militantes montoneros Eduardo Pereyra Rossi y Osvaldo Cambiaso. Y por último, Costanzo aportó un dato clave para lograr la restitución de la identidad de Sabrina Gullino Valenzuela Negro, hija de Raquel Negro y Tulio Valenzuela. Sabrina nació, junto con su hermano mellizo, en cautiverio en el Hospital Militar de Paraná como sobrina del general Leopoldo Galtieri. Según declaró Costanzo, en la etapa procesal de este juicio, que de acuerdo a lo que le contaron Pagano y Amelong el varón había nacido muerto y a la nena la 
dejaron en un convento en Rosario. Este dato se cruzó otra información disponible en la causa por la identidad de los hijos de Raquel Negro y Tulio Valenzuela y se pudo restituir la identidad de Sabrina.

\section{¿Qué Sucede Cuando los Represores Hablan?}

Ahora bien, el contenido de las declaraciones de Costanzo, el lugar en el que se produjeron y los efectos simbólicos y materiales que provocaron, permiten plantear un conjunto de interrogantes respecto del lugar que adquiere la palabra de los represores en los procesos de memoria y en los planos de verdad que la circulación pública de esta palabra condensa. Para avanzar en este análisis, es necesario hacer un desplazamiento del análisis de los dichos de Costanzo a las repercusiones y efectos de sus palabras entre los actores que fueron parte del proceso judicial en Rosario. Se trata de identificar el modo en que fueron escuchados e interpretados esos dichos, el carácter y el sentido que les otorgó y las respuestas y reacciones que generaron entre las querellas, la fiscalía, las victimas, así como las apreciaciones sobre la subjetividad del represor que la posición de enunciación de Costanzo despertó.

Como ya se mencionó, las declaraciones de Costanzo fueron excepcionales en comparación con lo dicho por otros represores en este y otros juicios por crímenes de lesa humanidad. De modo tal que, frente al silencio corporativamente mantenido, la sala de audiencias del Palacio de Tribunales de Rosario - y tal como sucede en otros juicios- se convirtió en el escenario donde se manifestó de manera directa, cara a cara, uno de los reclamos históricos de las víctimas y de los organismos de Derechos Humanos: el de Verdad. Cuando los imputados culminaron sus alegatos finales, los familiares y militantes de organismos de derechos humanos que se encontraban entre el público comenzaron a gritar y mostrar carteles que decían “¿dónde están los compañeros?” hasta que el tribunal ordenó desalojar la sala. Lo que se escenificó esa tarde en la sala de audiencias es la sustracción de un saber que el crimen de desaparición produce: que pasó exactamente con cada uno de los desaparecidos, en qué circunstancias fueron asesinados, quiénes son los responsables, dónde están sus restos y, también, dónde están los niño/as apropiado/as (JELIN, 1995).

Las declaraciones de Costanzo re-actualizaron esta expectativa pero también mostraron que, si bien se trata de un reclamo compartido entre sobrevivientes, familiares, organismos de derechos humanos y querellas, fueron divergentes las acciones para satisfacerlo. Los posicionamientos ético y políticos y las estrategias jurídicas no fueron homogéneas al respecto, aunque también existieron consensos que reivindicaron posturas 
innegociables de conjunto. En términos analíticos, el plano jurídico remite al modo en que fueron valorados los dichos de Costanzo para la construcción de la prueba judicial y la formulación de las acusaciones; el plano político involucra las luchas por la construcción del sentido sobre lo ocurrido durante el terrorismo de Estado y quién es el actor autorizado para expresarlo públicamente; y el plano ético consigna cómo posicionarse frente a lo que los represores hicieron en el pasado y ante lo que dicen y hacen en el presente.

En el plano jurídico, la construcción de la prueba implicó estrategias distintas respecto de la valoración de los dichos de Costanzo. Lo que se puso en juego fue la decisión de demostrar la existencia de los delitos y de responsabilidades penales de los imputados utilizando las declaraciones de un represor. Si bien esto tiene una resolución jurídica en función de legislación penal y procesal, no fue una cuestión meramente técnica para los equipos de abogados querellantes ni para la fiscalía. El trasfondo de las diferencias en el modo en que debía ser tratada la palabra de Costanzo remite no solo a cómo entienden estos actores, dentro y fuera de la escena judicial, la noción de verdad sino también al problema ético-político de dar lugar a los dichos de un represor, por qué hacerlo y de qué manera.

La querella de la Liga Argentina por los Derechos del Hombre (LADH) y de Familiares de Desaparecidos y Detenidos por Razones Políticas (Familiares) tuvo una actitud reticente ante la posibilidad de conformar prueba con las declaraciones de Costanzo y de considerarlas ciertas. ${ }^{37}$ Al respecto, sostenía la abogada querellante Gabriela Duruty

Si hay algo que se prueba solo con la declaración de Costanzo no nos alcanza. Cada cosa tratábamos de refrendarla con otro tipo de prueba, que no estuviera nunca sostenida solamente por los dichos de Costanzo y de ninguno de los repressores (Entrevista realizada el 16 de julio de 2016).

Al igual que esta querella, las otras partes acusatorias no construyeron sus alegatos a partir y exclusivamente de los dichos de Costanzo. En esta línea, la querella de H.I.J.O.S. tomó sus declaraciones como indicios sobre los que trabajar, los cuales junto con otras pruebas se lograron confirmar. ${ }^{38}$ Nadia Schujman, abogada de esa querella sostenía en un entrevista que, "las cosas que resultaban más inverosímiles con el tiempo y junto con otros pruebas resultaron que eran reales". Sin embargo, respecto de los hechos que solo se reconstruían a partir de las declaraciones de Costanzo, esta querella reconocía un aporte

37 LIDH es un organismo de derechos humanos fundado en 1937 históricamente vinculado al Partido Comunista y Familiares surgió, en Córdoba y en Buenos Aires, en 1976 frente a la necesidad de conocer el paradero de sus seres queridos desaparecidos.

38 La agrupación Hijos por la Identidad y la Justicia contra el Olvido y el Silencio (H.I.J.O.S.) surge hacia 1995 renovando el escenario de las luchas por la memoria con nuevas modalidades de lucha como el escrache los represores en sus domicilios y lugares de trabajo. Esta agrupación reúne a hijos/as de desaparecidos, asesinados y exiliados. 
sustantivo puesto se trataba de hechos cuyos detalles solo se pudieron conocer a través de su relato.

La noche de los homicidios, la puesta en escena de los homicidios, según él, que los cuerpos los arrojaron a la Bahía de Samborombón, es algo que podemos conocer solo por él (Entrevista a Nadia Schujman, 17 de julio de 2016).

Ambas estrategias jurídicas permiten observar los diversos planos que tiene la noción de verdad. Tales planos adquieren una espesura densa y problemática con la circulación pública de la palabra de un represor que rompió el "pacto de silencio". En primer lugar, las querellas reconocen un primer nivel de indicio en la palabra del represor, es decir, sus dichos constituyen una pista, una señal, que requiere de un trabajo cuidadosa de cotejo, revisión e investigación de parte de quién la escucha y lo toman como un rastro sobre el actuar y poder conocer lo sucedido.

En segundo lugar, los indicios se convierten en información valiosa cuando se completan, cotejan y confirman con otros datos que surgen de otras pruebas, de fuentes testimoniales, de hechos jurídicamente probados en otros juicios o demostrados en el informe de la CONADEP. Como una primera hipótesis respecto de los objetivos de este artículo, podríamos afirmar que la palabra de los represores responde a la demanda histórica de Verdad de los familiares, de las víctimas y de los organismos de Derechos Humanos cuando un conjunto de mediaciones la incorporan a una trama de relatos social e institucionalmente consignada como verdadera.

Pero, ¿qué sucedió durante el juicio cuando los indicios brindados por Costanzo no pudieron ser comprobados con otras pruebas, porque se trataba de una información que solo él, como represor, conocía? Aquí las valoraciones sobre los dichos se corren hacia un terreno más controvertido: la interrogación sobre las condiciones que hacen que la palabra de un represor pueda ser veraz por sí misma. Las estrategias jurídicas utilizadas para lidiar con esta cuestión fueron de tres tipos. Primero, se buscó demostrar la totalidad de los dichos eran veraces puesto que se comprobó que no mintió respecto de los hechos que sí fueron probados. Segundo, se trató de evaluar como creíbles las afirmaciones que no pudieron ser probadas con otros testimonios porque fueron sostenidas en el tiempo -el imputado venía repitiendo el mismo relato desde la entrevista de Rosario/12 en junio de 1992-. Y tercero, la fiscalía procuró señalar que Costanzo no tenía un interés mendaz o lucrativo cuando hablaba ni que estaba loco o tenía problemas psicológicos como buscaban demostrar las defensas de los otros co-imputados. Estas estrategias muestran un deslizamiento que va de la consideración sobre los dichos a la evaluación de la conducta y las intenciones del represor (si era mentiroso, si buscaba dinero) y de las acciones 
realizadas en el pasado a su comportamiento en el tiempo presente (si mostró una voluntad sostenida de hablar). De modo tal que las apreciaciones sobre la subjetividad del represor que se desplegaron en el juicio atienden no sólo a lo que ha cometido en el pasado sino también a lo que dice y hace en el tiempo presente (en los diversos presentes) respecto de ese pasado.

Ahora bien, estas estrategias jurídicas se apoyaban, a su vez, en diversos modos de posicionarse políticamente frente a la palabra y a la persona del represor. Todas las partes acusatorias valoraban los dichos de Costanzo cómo de quién venía: un represor. Y esto implicaba hacer frente al problema político que atraviesa las luchas por la memoria en Argentina, el dilema de autorizar como socialmente legítima la voz de los asesinos cuando ellos se han ocupado sistemáticamente de negar, ocultar, justificar, relativizar e, incluso, reivindicar los atrocidades cometidas (GONZÁLEZ LEEGSTRA, 2012). Sin embargo, las valoraciones a este respecto tampoco fueron homogéneas.

La querella de la LADH y de Familiares consideraba negativamente los dichos de Costanzo por la condición de represor del declarante y circunscribía su agencia a las acciones profundamente violatorias de la dignidad de las personas cometidas durante el terrorismo de Estado. De allí que Costanzo no era, para esta querella, "diferente" al resto de los imputados sino que tan solo era un represor que ponía en juego "una estrategia diferente". Al respecto, Gabriela Durruty sostenía,

[...] que alguna de las cosas que él haya dicho haya servido para el proceso de memoria, verdad y justicia no lo convierte en nada más que en eso en un tipo que tenia algún dato para tirar y que lo tenia porque participó.

La querella de H.I.J.O.S., en cambio, destacaba el contenido de información que los dichos de un represor que había roto el "pacto de silencio" podían contener y aportar. Mientras para la primera querella la condición del represor se imponía sobre sus dichos puesto que su conducta en tiempo presente era una continuidad de sus acciones pasadas, para la segunda -aunque no minimizaba ni relativiza que Costanzo era un miembro del grupo de tareas- le otorgaba valor a su palabra y producía un efecto de desacople entre lo que el represor hizo en el pasado -y lo que convierte en asesino- y lo que el represor puede hacer en el presente -que un asesino pueda hablar de lo que hizo y romper el pacto de silencio.

Estos modos de posicionarse jurídica y políticamente tienen implicancias en el plano ético. La experiencia humana de escuchar y prestar oído a los represores encierra un carácter dilemático para los actores involucrados en el juicio. Para la querella de la LADH y Familiares, la dificultad mayor residía justamente en "escuchar a estos tipos", mientras que 
para la querella de H.I.J.O.S. "no se podía desechar nada". Mientras la primera encontraba en la escucha un dilema ética, la otra asumía como una obligación también ética tomar como indicio lo que decía el represor para trabajar "seriamente" sobre la información que brindaba. Estas posturas surgían del modo en que Costanzo se vinculaba con los hechos que describía, esto es, con el lugar de enunciación que asumió cuando tomó la palabra. El hecho de posicionarse subjetivamente como "testigo y denunciante" de los actos aberrantes de los que fue parte, y no como el responsable además de no mostrar ningún signo de arrepentimiento, muestran el carácter performativo de la palabra de los represor y el carácter éticamente controversial que adquiere cuando habla.

Entre los efectos materiales y simbólicos de los dichos de Contanzo se puede mencionar que, si bien todas las partes acusatorias pidieron cadena perpetúa para Costanzo apoyadas en la convicción compartida de que Costanzo haya hablado no lo hacía menos represor y ni minimizaba los delitos por los que estaba imputado, sin embargo, una de las querellas solicitaron se le otorgue el beneficio de la prisión domiciliaria por el hecho de haber colaborado y porque su vida correría riesgos en caso de ir a cárcel. ${ }^{39}$ También algunos sobrevivientes y víctimas hicieron público un reconocimiento al hecho de que Costanzo hablase, pero mantuvieron el pedido de cadena perpetua. Tal fue el caso de Adriana Arce, sobreviviente de la Fábrica Militar de Armas y querellante en la causa, quién dijo durante su declaración testimonial:

\begin{abstract}
Lamentablemente los pactos de silencio que hay entre las fuerzas de seguridad han impedido que lleguemos a la verdad y en este sentido sí, quiero destacar la hombría que ha tenido el Sr. Costanzo (y lo señala) de romper ese pacto porque nos ayuda a llegar a la verdad y ejercer la justicia.
\end{abstract}

\title{
Conclusiones
}

A partir de lo expuesto y a modo de cierre, quiero abordar una cuestión fundamental para los procesos memoriales en Argentina: los diversos planos de enunciación de la verdad sobre el pasado que la circulación de la palabra de los represores permite pensar y problematizar, en relación con los diversos contextos en lo que se produce, es decir, períodos de impunidad o en el marco de los juicios por crímenes de lesa humanidad.

Un primer plano es el de la demanda de Verdad históricamente levantada por los organismos de derechos humanos, las víctimas y los familiares. Este reclamo no puede

39 Mientras la querella de Adriana Arce solicitó expresamente se conceda el arresto domiciliario a Costanzo, la querella de H.I.J.O.S. que históricamente se opone a los arrestos domiciliarios de los represores levantando la consigna "Cárcel común y efectiva", mantuvo luego de finalizado el debate oral una posición de no cuestionamiento a este beneficio cuando el tribunal se lo otorgó. 
reducirse al sentido estrictamente literal de sus formulaciones, esto es, a esa información vital e imprescindible que, sin duda, solo poseen los represores. Los debates que se produjeron a propósito de los dichos de Costanzo muestran que, si bien se trata de un reclamo por información, encierra otros elementos que exceden lo literal. Se trata de una demanda ética de reconocimiento del carácter horroroso de los hechos que cometieron, una búsqueda de verdad más profunda que excede sus especificaciones factuales, aunque no podría elaborarse sin ellas (OBERTI; PITTALUGA, 2016, p. 11).

Un segundo plano es el de la información brindada por los represores en los escasísimos casos en los que han hablado. Se trata de una información que tiene un carácter indiciario pero que adquiere el status de verdad cuando, luego de ser escuchada, los victimas, los familiares, los organismos de derechos humanos y la sociedad en su conjunto cuentan con mecanismos institucionales para sopesarla, investigarla, chequearla. La palabra de los represores adquiere diversas valoraciones según los contextos en los cuales ha sido enunciada. En el marco de la Justicia, a diferencia de la palabra que se produjo en los medios de comunicación en contextos de impunidad, es parte de un proceso institucional que coloca a quién escucha en una posición en la que cuenta con capacidad de respuesta, de acción y argumentación sobre lo dicho. En cambio, cuando la palabra se pronuncia y circula en los medios de comunicación en contextos de impunidad las víctimas quedan desprotegidos frente los dichos de los represores y estos cuentan con las condiciones para reivindicar o negar lo sucedido. En los juicios, las víctimas y familiares pueden, de mano de sus patrocinadores, preguntar y contestar, pedir mayores detalles, pero sobre todo, en el caso de que los imputados no quieran responder preguntas, y pueden argumentar más tarde en el momento de los alegatos y sopesar la información para construir prueba. Incluso pueden -como hizo Adriana Arce- hacer público un reconocimiento hacia el represor que ha hablado, reponer algo de su humanidad enajenada sin haber abdicado en su derecho de Justicia.

Un tercer plano surge los procesos institucionales y colectivos de construcción de la verdad. Desde la dictadura, los organismos de derechos humanos no sólo buscaron esclarecer lo ocurrido con los desaparecidos sino que llevaron adelante "una práctica activa de producción de verdad" (JELIN, 2017, p. 112) Esta construcción se apoya y completa con las investigaciones realizadas por la CONADEP y por las actuaciones judiciales (Juicio a los Ex Comandantes, juicios por la verdad y juicios por crímenes de lesa humanidad) que reunieron diversos medios de prueba. Este proceso, de más 30 años, ha permitido la configuración no sólo de una verdad sobre los hechos que la retira del terreno de lo meramente opinable; sino también de las significaciones centrales sobre el terrorismo de 
Estado a través de los cuales se pueden sustentar ciertas representaciones colectivas que no pueden ser ni negadas ni minimizadas por los dichos maniqueos de ningún represor sin generar un fuerte rechazo social.

Estos tres planos, históricos y contingentes, que adquirió el sentido de la verdad en los procesos memoriales en la Argentina, otorgándole una densidad social y política a esta categoría, nos permite revisar críticamente esa tendencia a esencializar la palabra de los perpetradores. Esta esencialización parte del supuesto de que una verdad más verdadera surgiría de su palabra y que esa verdad es condición natural de la posición del victimario y que si esa verdad no se produce se debe a que los instrumentos judiciales lo impiden. Como vimos, las nociones de verdad que sostienen los actores implicados en la causa Guerrieri I muestran que está un nivel que involucra el hallazgo de datos, por más valiosos que puedan ser, que conocen los represores, pero que no se reduce a ello. Y además, no se trata de dar como existente una verdad guardada por los represores sino de situar esa información en un proceso más perdurable y profundo que será veraz siempre y cuando se acople el conjunto de verdades ya probadas, que tienen la cualidad de apoyarse unas con otras, fruto de un proceso de más de tres décadas que ha permitido, a pesar del silencio de los represores, tener bastante certezas sobre lo qué pasó durante el terrorismo de Estado en la Argentina.

\section{Referências}

ÁGUILA, Gabriela (ed.). Territorio ocupado: la historia del Comando del II Cuerpo de Ejército en Rosaior (1960-1990). Rosario: Museo de la Memoria, 2018.

ÁGUILA, Gabriela. Dictadura, represión y sociedad en Rosario, 1976/1983: un estudio sobre la represión y los comportamientos y actitudes sociales en dictadura. Buenos Aires: Prometeo, 2008.

BONASSO, Miguel. Recuerdo de la muerte. Buenos Aires: Planeta, 2001.

CALVEIRO, Pilar. Poder y desaparición: los campos de concentración en Argentina. Buenos Aires: Colihue, 1998.

CELS - CENTRO DE ESTUDIOS LEGALES Y SOCIALES. Terrorismo de Estado: 692 responsables. Buenos Aires: CELS, 1986.

CRENZEL, Emilio. La historia política del Nunca Más: la memoria de los desaparecidos en la Argentina. Buenos Aires: Siglo XXI, 2008.

FELD, Claudia. El imposible "debate" entre víctimas y victimarios: notas sobre las declaraciones televisivas de Miguel Etchecolatz (1997). Rúbrica Contemporánea, Barcelona, v. 3, n. 9, p. 77-101, 2016. Disponible en: https://revistes.uab.cat/rubrica/article/view/v5-n9feld/115-pdf-es. Acceso en: 15 mayo 2018. 
FELD, Claudia. La construcción del "arrepentimiento": los ex represores en la televisión. Entrepasados, Buenos Aires, n. 20-21, p. 35-53, 2001.

GONZÁLEZ LEEGSTRA, Cintia. "No le vamos a dar voz a los represores": (des)autorizaciones y (des)legitimaciones en las audiencias del juicio a Etchecolatz. Sociohistórica, La Plata, n. 29, p. 87-105, 2012. Disponible en: http://www.sociohistorica.fahce.unlp.edu.ar/article/view/shn29a04/2442. Acceso en: 24 mayo 2018.

GRÜNER, Eduardo. La cólera de Aquiles: una modesta proposición sobre la culpa y la vergüenza. Conjetural, Buenos Aires, n. 31, p. 17-41, 1995.

JANKÉLÉVITCH, Vladimir. La mala conciencia. Ciudad de México: Fondo de Cultura Económica, 1987.

JELIN, Elizabeth. La lucha por el pasado: cómo construimos la memoria social. Buenos Aires: Siglo XXI, 2017.

JELIN, Elizabeth. La política de la memoria: el movimiento de Derechos Humanos y la constitución de la democracia en la Argentina. In: ACUÑA, Carlos H. Juicio, castigos y memorias: derechos humanos y justicia en la política argentina. Buenos Aires: Nueva Visión, 1995. p. 101-146.

OBERTI, Alejandra; PITTALUGA, Roberto. Apuntes para una discusión sobre la memoria y la política de los años 60/70 a partir de algunas intervenciones recientes. Sociohistórica, La Plata, $\quad$ n. $38, \quad 2016.2$ Disponible http://sedici.unlp.edu.ar/bitstream/handle/10915/59757/Documento_completo.pdfPDFA.pdf? sequence=1\&isAllowed=y. Acceso en: 27 mayo 2018.

SALVI, Valentina. "Entelequia", "enmascaramiento" y "disimulo": las últimas declaraciones de Videla sobre los desaparecidos (1998-2012). Revista Rúbrica Contemporánea, Barcelona, v. 5, n. 9, p. 103-122, 2016. Disponible en: https://revistes.uab.cat/rubrica/article/view/v5-n9salvi/pdf_9. Acceso en: 12 mayo 2018.

SALVI, Valentina. De vencedores a víctimas: memorias militares sobre el pasado reciente en la Argentina. Buenos Aires: Biblios, 2012.

SALVI, Valentina. El universo de los represores: una mirada desde los estudios de memoria en la Argentina. In: JARA, Daniela; AGUILERA, Carolina (ed.). Pasados inquietos: los desafíos de la memoria pública de los perpetradores de violaciones de DD.HH. y crímenes de lesa humanidad en Argentina y Alemania. Santiago de Chile: Museo de la Memoria y los Derechos Urbanos, 2017. p. 34-56.

SCOTT, Joan Wallach. Experiencia. La Ventana, Guadalajara, n. 17, p. 42-73, 2001.

VARSKY, Carolina. El testimonio como prueba en procesos penales por delitos de lesa humanidad: algunas reflexiones sobre su importancia en el proceso de justicia argentino. In: CELS - CENTRO DE ESTUDIOS LEGALES Y SOCIALES; CIJT - CENTRO INTERNACIONAL PARA LA JUSTICIA TRANSICIONAL. Hacer justicia: nuevos debates sobre el juzgamiento de crímenes de lesa humanidad en la Argentina. Buenos Aires: Siglo XXI, 2011. 